\title{
Current techniques for management of transverse displaced olecranon fractures
}

\author{
Anniek den Hamer ${ }^{1}$ \\ Maarten Heusinkveld ${ }^{1}$ \\ Willeke Traa ${ }^{1}$ \\ Pim Oomen ${ }^{1}$ \\ Francesco Oliva² \\ Angelo Del Buono 3 \\ Nicola Maffulli ${ }^{4}$
}

1 Department of Biomedical Engineering, Eindhoven University of Technology, Eindhoven, Netherlands

2 Department of Orthopaedics, Tor Vergata University, Rome, Italy

4 Department of Orthopaedic and Trauma Surgery Hospital Antonio Cardarelli, Campobasso, Italy

${ }^{4}$ Queen Mary University of London, Centre for Sports and Exercise Medicine, Mile End Hospital, Mann Ward, London, England; Department of Musculoskeletal Disorders, University of Sabino School of Medicine and Surgery, Sabino, Italy

Corresponding author:

Nicola Maffulli

Queen Mary University of London, Centre for Sports and Exercise Medicine, Mile End Hospital, Mann Ward 275 Bancroft Road, London E1 4DG, England E-mail: n.maffulli@qmul.ac.uk

\section{Summary}

Background: displaced transverse fractures of the olecranon are the most common fractures occurring in the elbow in adults that requires operative intervention.

Methods: a literature search was performed on PubMed, Web of Science, Science Direct/Scopus, Google Scholar and Google using the keywords 'olecranon', 'fracture', 'internal fixation' and 'tension band wiring', with no limit for time or restrictions to language.

Results: thirty-one clinical articles were selected: 20 retrospective studies, 9 prospective cohort studies, and 2 randomized control trials. The CMS ranged from 18 to 66 (mean 41.68): overall, the quality of the studies was poor, and no moderate or good quality studies were found. The mean follow-up was 46.7 months (range 1 to 350 months). Several complications occurred after surgery: prominent hardware, skin breakdown, wire migration and infections oc- curred frequently. Removal of the hardware was required in $\mathbf{4 7 2}$ patients, usually after complaints, but also removal was routinely undertaken.

Conclusions: tension band wiring is still the most widely applied method to operatively manage olecranon fractures, with the transcortical method of using $\mathrm{K}$-wires the most satisfactory. Plate fixation is a good alternative as complications are minimal. Other techniques using absorbable sutures are less investigated, but are promising, especially in children.

KEY WORDS: displaced olecranon fractures, outcomes, surgery, systematic review.

\section{Introduction}

Displaced transverse fractures of the olecranon are the most common fractures occurring in the elbow in adults that requires operative intervention ${ }^{1-4}$. Olecranon fractures are mostly caused by motor vehicle crashes, falls from a height $(>2 m)$, simple falls or a direct blow to the elbow and are commonly observed orthopedic injuries in the emergency room ${ }^{2,5-7}$. Fracture patterns can be assessed by different classification methods, with the Mayo most commonly used. This method takes into account the degree of the fracture displacement, the stability of the elbow joint and if the fracture is comminuted. Mayo Type I fractures are undisplaced fractures; these are uncommon and can be managed conservatively. However, most olecranon fractures are displaced (Type II and III) and operative treatment is recommended. Type II are displaced, stable fractures where the displacement is more than three millimeters, the collateral ligaments are intact and the forearm is stable in relation to the humerus, this type can be non-comminuted (Type IIA) or comminuted (Type IIB). Type III fractures are displaced and unstable, this fracture is most difficult to treat and has the poorest prognosis ${ }^{8}$. Various fixation techniques have been used for olecranon fractures nowadays, including tension-band wiring (TBW) in different configurations $2,9-11$ combined with Kirschner wires (Kwires $^{1,6,12}$, cancellous screws ${ }^{13}$, plate (PF) and screw fixation $^{7,14-16}$, Netz pins ${ }^{17}$, biodegradable pins ${ }^{18}$ or absorbable sutures ${ }^{19,20}$. The most common method is TBW21, first described by Weber and Vasey in $1963^{22}$. TBW relies on open reduction and internal fixation. TBW is recommended for transverse fractures by the Arbeitsgemeinschaft für Osteosynthesefragen (AO) and is the most commonly used operative technique for internal fixation of olecranon fractures $2,3,12,23$. With TBW, a high 
union rate and good functional recovery have been reported ${ }^{3,10,24}$. The technique consists of two K-wires inserted from the proximal aspect of the olecranon along the long axis of the ulna (intramedullary), and a tensionband wire loop. This technique is simple and effective in transferring the forces that are produced by the pull of the triceps mechanism into forces that induce compression of the fracture. In this way, early elbow motion is allowed, preventing elbow stiffness ${ }^{1}$.

However, complications can arise from TBW, the most common being prominence of the K-wires, which causes skin irritation ${ }^{13,25,26}$. Skin penetration and infections are also observed, and delayed union can occur because of this infection. Migration of the wires can cause cartilage injury, soft-tissue problems and local pain, and therefore re-interventions may be necessary. Loss of motion, extension, and flexion are usually observed by the patients and hard-ware removal is typically requested $2,12,15,21,24,25$. Other complications may reveal themselves at clinical examination during follow up, for example the development of osteoarthritis ${ }^{1,2}$. Therefore, adaptations and improvements have been made to overcome these complications when treating transverse olecranon fractures $2,4,6,10,27$.

We performed a systematic review of literature to ascertain which techniques are successfully used to manage displaced transverse olecranon fractures. An overview is produced of the surgical techniques and materials used, and evaluation of the intervention is considered.

\section{Methods}

\section{Literature search and data extraction}

A literature search was performed on 18.12.2012 using PubMed, Web of Science, Science Direct/Scopus, Google Scholar and Google. The latter was included to find studies not published in peer reviewed journals, but presented in lectures or congresses. The databases were searched using the keywords 'olecranon', 'fracture', 'internal fixation' and 'tension band wiring'. No time limit or restrictions to language were applied. Two reviewers assessed the abstracts of all studies identified by the initial search and excluded non-relevant studies. We excluded studies performed on different bones, different parts of the bone (e.g. the shaft of the ulna) and other types of fracture (e.g. mul- ti fragmentary). Subsequently elimination was made considering the titles of the obtained studies. All titles not relevant to the subject at hand were excluded. Articles in Chinese, Korean and Turkish were excluded, as were case reports, reviews, letters to editors, proceedings and book chapters. Four studies reported on animals, two of them reported on horses and two on dogs, and will not be discussed in this systematic review. The process of literature search is shown in Figure 1. Finally a subdivision was made of biomechanical and clinical studies. Only clinical studies were taken into account. Eventually, 31 studies remained; full text articles were obtained of these remaining studies.

\section{Quality Assessment}

Two reviewers independently scored the quality of the remaining studies using the Coleman Methodology Score (CMS) (Tab. 1). The Coleman scoring system analyzes whether the quality of the studies reviewed in systematic reviews is accurate and reproducible. This method has been used successfully for several years $^{21}$. It assesses the methodology using 10 pre-established criteria, giving a total score between 0 and 100. A score approaching 100 indicates that the study has a robust design and largely avoids chance, various biases' or confounding factors. A score $>85$ is considered excellent, $70-84$ good, 50-69 moderate, and $<50$ poor. Two investigators independently scored the quality of the studies independently. Each investigator scored the quality of the studies once. Where differences were encountered, agreement was achieved by consensus. SPSS version 20 for Windows (SPSS Inc., Chicago, IL, USA) was used to analyze the data.

\section{Results}

\section{Studies}

Of 1483 studies, 63 studies were relevant. From these, 31 clinical articles were selected. The articles were published between 1982 and 2012. From the 31 articles addressed, 20 were retrospective studies, 9 were prospective cohort studies and 2 randomized control trials. The studies were performed in 17 different countries; Austria (1), Australia (1), Denmark (2),

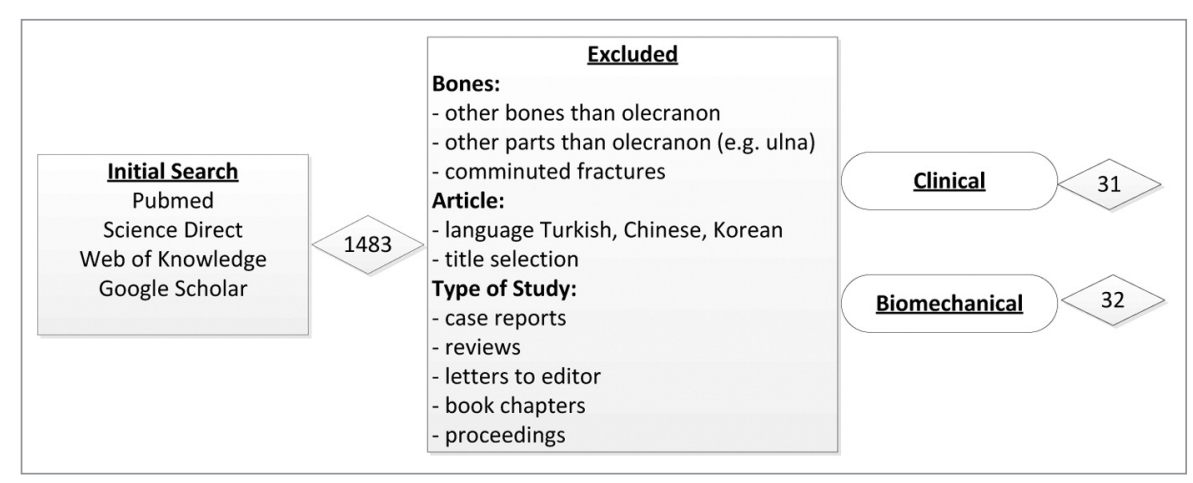

Figure 1. Literature search and application of exclusion and inclusion criteria gave 31 articles when an initial search with the keywords 'olecranon', 'fracture', 'internal fixation' and 'tension band wiring' was performed. 
Table 1. Criteria used to compute the Coleman Methodology Score (CMS) for clinical studies reporting transverse olecranon fractures.

\begin{tabular}{|c|c|c|c|}
\hline Score\# & Part A - Only one score to be given for each of th & seven sections & Score \\
\hline 1 & Study size - number of olecranon fractures included & $>60$ & 10 \\
\hline & & $41-60$ & 7 \\
\hline & & $20-40$ & 4 \\
\hline & & $<20$, not stated/unclear & 0 \\
\hline 2 & Mean follow-up (months) & $>24$ & 5 \\
\hline & & $12-14$ & 2 \\
\hline & & $<12$, not stated/unclear & 0 \\
\hline 3 & Number of different surgical procedures included & One surgical procedure only & 10 \\
\hline & in each reported outcome & More than one surgical procedure, & 7 \\
\hline & & $\begin{array}{l}\text { Not stated, unclear or }<90 \% \text { of subjects } \\
\text { undergoing the procedure }\end{array}$ & 0 \\
\hline 4 & Type of study & Randomized control trial & 15 \\
\hline & & Prospective cohort study & 10 \\
\hline & & Retrospective cohort study & 0 \\
\hline 5 & Diagnostic certainty (use of preoperative ultrasound, & In all & 5 \\
\hline & MRI, postoperative histopathology & In $>80 \%$ & 3 \\
\hline & to confirm diagnosis) & In $<80 \%$, no certainty or unclear & 0 \\
\hline 6 & Description of surgical procedure given & $\begin{array}{l}\text { Adequate (technique stated and necessary } \\
\text { details of that type of procedure given) }\end{array}$ & 5 \\
\hline & & Fair (technique only stated with elaboration) & 3 \\
\hline & & Inadequate, not stated or unclear & 0 \\
\hline 7 & Description of postoperative rehabilitation & Well described with $>80 \%$ of patients complying & 10 \\
\hline & & Well described with $60-80 \%$ of patients complying & 5 \\
\hline & & Protocol not reported or $<60-80 \%$ complying & 0 \\
\hline
\end{tabular}

Part B- scores may be given for each option of the three sections if applicable

\begin{tabular}{|c|c|c|c|}
\hline 1 & Outcome criteria & $\begin{array}{l}\text { Outcome measures clearly defined } \\
\text { Timing of outcome assessment clearly stated } \\
\text { (at best outcome after surgery or at follow up) } \\
\text { Use of outcome criteria that has reported } \\
\text { good reliability } \\
\text { Use of outcome with good sensitivity }\end{array}$ & $\begin{array}{l}2 \\
2\end{array}$ \\
\hline 2 & Procedure for assessing outcomes & $\begin{array}{l}\text { Subjects recruited (results not taken from } \\
\text { surgeon's files) } \\
\text { Investigator independent of surgeon } \\
\text { Written assessment } \\
\text { Completion of assessment by subjects } \\
\text { themselves with minimal investigator assistance }\end{array}$ & $\begin{array}{l}4 \\
3 \\
3\end{array}$ \\
\hline 3 & Description of subject selection process & $\begin{array}{l}\text { Selection criteria reported and unbiased } \\
\text { Recruitment rate reported }>80 \% \\
\text { Recruitment rate reported }<80 \% \\
\text { Egligible subjects not included in the study } \\
\text { satisfactorily accounted for a } 100 \% \text { recruitment }\end{array}$ & $\begin{array}{l}5 \\
5 \\
3 \\
5\end{array}$ \\
\hline
\end{tabular}

China (1), Egypt (1), France (1), Germany (1), Great Britain (1), Greece (1), Ireland (2), Israel (1), Netherlands (3), Singapore (1), Spain (1), Sweden (3), Taiwan (3), United States of America (7).

\section{Quality Assessment}

The CMS ranged from 18 to 66 (mean 41.68, SD 12.78) (Tab. 2): overall, the quality of the studies was poor, and no moderate or good quality studies were found.

\section{Demographic data and postoperative details}

From the 31 studies selected that discussed humans, only 2 considered only displaced transverse olecranon fractures ${ }^{12,28}$, all the others included several types of fractures. The studies published between 1982 and 2012 discussed between 6 and 118 fractures per article. Within the 31 studies, 1284 patients were treated with 192 patients classified as having a displaced transverse fracture of the olecranon. Overall, patients were between 1 and 97 years old, with a 
Table 2. Overview selected articles with CMS score, number of fractures discussed in articles, male/female ratio, average age, techniques performed and follow-up time.

\begin{tabular}{|c|c|c|c|c|c|c|}
\hline Article & $\begin{array}{l}\text { CMS } \\
\text { score }\end{array}$ & $\begin{array}{l}\text { \# Fractures } \\
\text { (Transverse/ } \\
\text { TypellA) }\end{array}$ & $\begin{array}{l}\text { Male/ } \\
\text { Female } \\
\text { ratio }\end{array}$ & $\begin{array}{l}\text { Average } \\
\text { Age }\end{array}$ & Technique & $\begin{array}{l}\text { Follow-up } \\
\text { (Average } \\
\text { in months) }\end{array}$ \\
\hline Ahmed $2008^{13}$ & 31 & 30 & $21 / 9$ & & TBW or intramedullary screw & \\
\hline Anderson $2007^{14}$ & 50 & $32(17)$ & $18 / 14$ & 54,20 & Elbow plate & 26.40 \\
\hline Arbes $2012^{29}$ & 20 & 15 & $11 / 4$ & 7,6 & $\begin{array}{l}\text { Kwire, TBW, absorbable } \\
\text { suture, plate }\end{array}$ & 14 \\
\hline Bailey $2001^{16}$ & 65 & $25(5)$ & $11 / 14$ & 44,00 & Reconstruction plate & 34 \\
\hline Chalidis $2008^{21}$ & 65 & $62(40)$ & $33 / 29$ & 48,60 & TBW, K-wire, Figure-of-8 & 98.40 \\
\hline Fan $1993^{11}$ & 24 & 118 & $79 / 39$ & 35,00 & $\begin{array}{l}\text { Olecranon screw, K-wire, } \\
\text { Rush pin }\end{array}$ & 28 \\
\hline Gicquel $2003^{30}$ & 34 & 6 & $4 / 2$ & 10,20 & & 12.00 \\
\hline Gortzak 2006 ${ }^{19}$ & 51 & 6 & $5 / 1$ & 9,75 & $\begin{array}{l}\text { K-wires, Absorbable tension } \\
\text { band suture (Vicryl } 2 \text { or DexonlI) }\end{array}$ & 13.00 \\
\hline Holdsworth $1984^{3}$ & 36 & 52 & $28 / 24$ & & $\begin{array}{l}\text { TBW, supplementary screws, } \\
1 / 3 \text { tubular plate }\end{array}$ & 24 \\
\hline Hope $1991^{18}$ & 38 & 24 & $17 / 7$ & & K-wires, Figure-of- 8 or PGA pin & \\
\hline Huang $2010^{6}$ & 53 & 77 & $45 / 32$ & & K-wires & 33.12 \\
\hline Hume $1992^{5}$ & 45 & $41(11)$ & & 30,90 & $\begin{array}{l}\text { TBW, K-wire, Figure-of- } 8 \text {, } \\
\text { or PF } 1 / 3 \text { tubular plate }\end{array}$ & 7,1 \\
\hline Jensen $1986^{26}$ & 25 & 53 & & 58,00 & TBW, K-wires & 38 \\
\hline Karlsson $2002^{10}$ & 50 & 67 & $26 / 41$ & & TBW or Figure-of-8 & 218.40 \\
\hline Karlsson $2002^{24}$ & 43 & 73 & $28 / 45$ & & $\begin{array}{l}\text { TBW, Figure-of-8, Rush pin, } \\
\text { Rissler peg }\end{array}$ & \\
\hline Koslowsky $2009^{27}$ & 59 & 12 & & 56,00 & $\begin{array}{l}\text { FFS system (new system using } \\
\text { fine threaded wires) }\end{array}$ & 28.60 \\
\hline Kuo $2011^{9}$ & 21 & $62(23)$ & $39 / 23$ & 39,00 & K-wire & \\
\hline Larsen $1991^{4}$ & 44 & $20(14)$ & $6 / 14$ & 64,00 & Adapted Netz pin & 4 \\
\hline Lindenhovius $2008^{7}$ & 41 & $20(3)$ & $13 / 7$ & 30,00 & $\begin{array}{l}\text { TBW, Figure-of- } 8, \mathrm{PF}, 1 / 3 \text { tubular } \\
\text { plate, dynamic compression plate }\end{array}$ & 216.00 \\
\hline Liu $2012^{31}$ & 55 & $62(50)$ & $30 / 32$ & 47,00 & Cable pin system (CPS) or TBW & 21 \\
\hline Low $1988^{32}$ & 36 & $32(13)$ & $22 / 10$ & 33,30 & TBW and K-wire & 8.2 \\
\hline Macko $1985^{25}$ & 31 & 20 & & 35,50 & K-wire, gauge tension wire & 13.5 \\
\hline $\begin{array}{l}\text { Mullett } 200012 \\
\text { (K-wire) }\end{array}$ & 35 & $80(80)$ & & 56,40 & K-wire & $39-43$ \\
\hline Mullet 2000 (TBW) 28 & 43 & $34(34)$ & & & K-wire & 42.00 \\
\hline Netz $1982^{17}$ & 49 & 10 & & 56,50 & TBW using Netz pins & \\
\hline Rommens $2005^{23}$ & 66 & $95(26)$ & $48 / 47$ & 47,70 & $\begin{array}{l}\text { TBW }(77), \text { TBW + lag screws (10), } \\
\text { TBW osteosynthesis proximal } \\
\text { radius (3), plate osteosynthesis (5) } \\
\text { nonoperatively (13) }\end{array}$ & \\
\hline Tejwani $2002^{15}$ & 39 & $20(8)$ & $13 / 7$ & 37,50 & posterior plating technique & 12.00 \\
\hline Linden $2011^{1}$ & 49 & $59(40)$ & $28 / 28$ & & K-wire, 18 gauge tension band & \\
\hline Villanueva $2006^{2}$ & 35 & $37(20)$ & $13 / 24$ & 63 & TBW, K-wire, Figure-of-8 & \\
\hline Wissing $1991^{20}$ & 27 & 36 & & & Vicryl nr 2.; K-wire & \\
\hline Wolfgang $1987^{33}$ & 32 & $41(20)$ & $27 / 18$ & 40,5 & TBW, K-wire, Figure-of-8 & \\
\hline
\end{tabular}


mean age of 41.7 (95\% Cl 35.3-48.1), with two studies not providing the relevant data 6,20 .

On average, the various studies included 23 men (range 4-79, 95\% Cl 16.1-30.0), and 19.4 women (range $1-47,95 \% \mathrm{Cl} 13.5-25.3$ ). Eight studies did not provide relevant data $5,12,17,20,25-28$.

The mean follow-up for all the patients was 46.7 months $(95 \% \mathrm{Cl} 20.5-72.9$, range 1 to 350 months). The cause of the injury was reported in 18 studies (shown in Tab. 3): in total, there were 358 high energy trauma episodes and 446 low energy, with high energy injuries described as falls from a height and motor vehicle accidents, while low energy traumas are stated as a simple fall, direct blows, or sports injuries.

\section{Outcome}

Union of the fractures was poorly described in the articles. Ten articles mentioned minimum and maximum time to union with a range from 2 to 12 months.

Several complications occurred after surgery (Tab. 6). Prominent hardware, skin breakdown, wire migration and infections occurred frequently. Removal of the hardware was required in 472 patients, usually after complaints, but also removal was routinely undertaken ${ }^{26}$.

Seven studies completely described the range of motion at follow-up, divided into ranges of flexion, extension, pronation and supination (Tab. 4). Furthermore, different scoring methods were used to describe the

Table 3. Causes of injury divided in High and Low Energy Trauma.

\begin{tabular}{|c|c|c|c|c|c|c|c|c|c|c|c|}
\hline \multirow[t]{3}{*}{ Author } & \multirow{3}{*}{$\begin{array}{l}\text { Injuries } \\
\text { Total }\end{array}$} & \multicolumn{3}{|c|}{ High Energy Trauma } & \multicolumn{6}{|c|}{ Low Energy Trauma } & \multirow{3}{*}{$\begin{array}{l}\text { Not } \\
\text { Described }\end{array}$} \\
\hline & & \multirow[t]{2}{*}{ Total } & \multicolumn{2}{|c|}{ Specification } & \multirow[t]{2}{*}{ Total } & \multicolumn{5}{|c|}{ Specification } & \\
\hline & & & $\begin{array}{l}\text { Motor } \\
\text { vehicle }\end{array}$ & $\begin{array}{l}\text { Fall } \\
\text { Height }\end{array}$ & & Blow & $\begin{array}{l}\text { Simple } \\
\text { Fall }\end{array}$ & Sport & Crushed & Bicycle & \\
\hline Fan $1993^{11}$ & 118 & 83 & 83 & & 35 & & & & & & \\
\hline Karlsson $2002^{10}$ & 67 & 31 & & & 33 & & & & & & 3 \\
\hline Karlsson $2002^{24}$ & 73 & 29 & & & 31 & & & & & & 3 \\
\hline Macko $1985^{25}$ & 20 & 16 & 6 & & 4 & 4 & 10 & & & & \\
\hline Huang $2010^{6}$ & 91 & 69 & 63 & 6 & 22 & & 22 & & & & \\
\hline Holdsworth $1984^{3}$ & 52 & 19 & 19 & & 27 & & 25 & 2 & & & \\
\hline Lindenhovius $2008^{7}$ & 20 & 15 & 8 & 6 & 5 & 2 & 1 & 2 & 1 & & \\
\hline Chalidis $2008^{21}$ & 62 & 24 & & & 38 & & 38 & & & & \\
\hline Villanueva 2006² & 37 & 3 & 2 & 1 & 34 & 3 & 30 & & & 1 & \\
\hline Wolfgang $1986^{33}$ & 45 & 20 & 20 & & 25 & 3 & 22 & & & & \\
\hline Bailey $2000^{16}$ & 25 & 2 & 2 & & 23 & & 20 & & & 3 & \\
\hline Mullett 2000 (TBW) $)^{28}$ & 34 & 6 & 6 & & 28 & 1 & 27 & & & & \\
\hline Ahmed $2008^{13}$ & 31 & 10 & 7 & 3 & 21 & 8 & 12 & & & & \\
\hline Hume $1991^{5}$ & 41 & 7 & 7 & & 34 & 19 & 15 & & & & \\
\hline Low $1988^{32}$ & 32 & 0 & & & 32 & 2 & 30 & & & & \\
\hline Liu $2012{ }^{31}$ & 62 & 23 & 23 & & 39 & & 34 & 5 & & & \\
\hline Arbes $2012^{29}$ & 15 & 1 & 1 & & 14 & & 14 & & & & \\
\hline
\end{tabular}

Table 4. Range of Motion.

\begin{tabular}{|c|c|c|c|c|}
\hline Article & Flexion & Extension & Pronation & Supination \\
\hline Anderson $^{14}$ & $120^{\circ} \pm 18.1$ & $13.5^{\circ} \pm 13.6$ & \multicolumn{2}{|c|}{ Pro/sup: $157.7^{\circ} \pm 29.2$} \\
\hline Bailey $^{16}$ & $128.5^{\circ} \pm 6.6$ & $8.5^{\circ} \pm 6.2$ & $76.7^{\circ} \pm 8.7$ & $67.2^{\circ} \pm 17.4$ \\
\hline Karlsson ${ }^{10}$ & $139.1^{\circ} \pm 1.3$ & $6.8^{\circ} \pm 1.4$ & $83.8^{\circ} \pm 1.5$ & $82.7^{\circ} \pm 1.9$ \\
\hline Karlsson 24 & $139^{\circ} \pm 1$ & $5^{\circ} \pm 1$ & $84^{\circ} \pm 1$ & $82^{\circ} \pm 29$ \\
\hline Koslowsky 27 & $\begin{array}{l}135.4^{\circ} \\
\left(\text { range } 130-140^{\circ}\right)\end{array}$ & $\begin{array}{l}1.4^{\circ} \\
\left(\text { range } 0-10^{\circ}\right)\end{array}$ & $\begin{array}{l}87.1^{\circ} \\
\left(\text { range } 80-90^{\circ}\right)\end{array}$ & $\begin{array}{l}85.4^{\circ} \\
\left(\text { range } 80-90^{\circ}\right)\end{array}$ \\
\hline Lindenhovius $^{7}$ & $134^{\circ} \pm 11$ & $12^{\circ} \pm 26$ & $70^{\circ} \pm 30$ & $81^{\circ} \pm 15$ \\
\hline Van der Linden 1 & $\begin{array}{l}\text { Short follow-up; } 133^{\circ} \pm 11 \\
\text { Long follow-up; } 141^{\circ} \pm 10\end{array}$ & $\begin{array}{l}\text { Short follow-up; } 15^{\circ} \pm 17 \\
\text { Long follow-up; } 14^{\circ} \pm 11\end{array}$ & $\begin{array}{l}\text { Short follow-up; } 78^{\circ} \pm 4 \\
\text { Long follow-up; } 78^{\circ} \pm 8\end{array}$ & $\begin{array}{l}\text { Short follow-up; } 78^{\circ} \pm 18 \\
\text { Long follow-up; } 78^{\circ} \pm 12\end{array}$ \\
\hline
\end{tabular}


outcome (Tab. 5). The MEPI and DASH score were the most commonly used. The remaining studies used other or subjective scores, which make them non-comparable. The Mayo Elbow Performance Index (MEPI) is one of the most commonly used elbow rating systems. This score takes into account pain,

Table 5. Assessment elbow function.

\begin{tabular}{|c|c|c|c|c|c|c|c|}
\hline Article & \# Fractures & $\begin{array}{l}\text { MEP } \\
\text { Total score }\end{array}$ & Excellent & Good & Fair & Poor & DASH \\
\hline Ahmed - screw ${ }^{13}$ & 15 & & 11 & 4 & 0 & 0 & \\
\hline Ahmed - TBW 13 & 15 & & 6 & 5 & 3 & 1 & \\
\hline Anderson ${ }^{14}$ & 32 & $89(55-100)$ & & & & & $25(0-72.5)$ \\
\hline Bailey $^{16}$ & 25 & 89 & 13 & 10 & 1 & 1 & $10 \pm 15.6$ \\
\hline Chalidis $^{21}$ & 62 & & 53 & & 6 & 3 & \\
\hline Fan $^{11}$ & 118 & & 38 & 36 & & & \\
\hline Lindenhovius $^{7}$ & 20 & $90 \pm 15$ & 13 & 4 & 2 & 1 & $8 \pm 14$ \\
\hline $\begin{array}{l}\text { Van der Linden } \\
\text { (short term) }^{1}\end{array}$ & 21 & $93 \pm 12$ & 15 & 5 & 0 & 1 & $12 \pm 16$ \\
\hline Villanueva $^{2}$ & 37 & $88(45-100)$ & 22 & 10 & 3 & 2 & $18(0-83)$ \\
\hline
\end{tabular}

Table 6. Complications and removal of hardware.

\begin{tabular}{|c|c|c|}
\hline Author & Complications & Removal in \# of patients \\
\hline Ahmed $2008^{13}$ & $\begin{array}{l}\text { TBW; prominent hardware, true wire migration (2), wound } \\
\text { infection (1). Screw; none }\end{array}$ & 1 (screw), 8 (TBW) \\
\hline Anderson $2007^{14}$ & Non union (2) & 3 \\
\hline Arbes $2012^{29}$ & $\begin{array}{l}\text { Reduction failure (5) due to; incomplete primary reduction (2), } \\
\text { secondary loss reduction (2), one K-wire did not fix fracture (1) }\end{array}$ & 15 \\
\hline Bailey $2000^{16}$ & Mild joint space narrowing (2), class I heterotopic ossification (2) & 5 \\
\hline Chalidis 2008 21 & Degenerative changes (30), infection, non union & $\begin{array}{l}51 \text { ( } 34 \text { still mild pain daily } \\
\text { life after removal) }\end{array}$ \\
\hline Fan $1993^{11}$ & Non union, wire migration & \\
\hline Gicquel $2003^{30}$ & & 6 (after 6 weeks) \\
\hline Gortzak $2006^{19}$ & & 6 (after 4 weeks) \\
\hline Holdsworth $1984^{3}$ & $\begin{array}{l}\text { No symptoms (38), pain only if knocked (9), occasional } \\
\text { spontaneous pain or clicking (4), infection (1), hypertrophic scars } \\
\text { (4), re-fracture twice in one patient (13 year old, osteogenesis } \\
\text { imperfecta refused stop playing football) }\end{array}$ & $\begin{array}{l}56 \% \text { removed average after } 6 \\
\text { months(half no complaints, } \\
\text { average age } 26 \text { years) }\end{array}$ \\
\hline Hope $1991^{18}$ & $\begin{array}{l}\text { Broken drill (1), infection K-wire (3), K-wire ectopic bone formed but } \\
\text { no dysfunction (1), PGA avulsion fracture avascular necrosis (1) }\end{array}$ & $\begin{array}{l}9 \text { (K-wire) due to too firmly } \\
\text { fixed wires }\end{array}$ \\
\hline Huang $2010^{6}$ & $\begin{array}{l}\text { K-wire migration, non union(1), wound infection; } 0 \% \text { (proximal), } \\
3.5 \% \text { (anterior), 3.8\% (distal) }\end{array}$ & $\begin{array}{l}33.3 \% \text { (proximal), } 10.7 \% \\
\text { (anterior), } 7.7 \% \text { (distal), } \\
\text { average after } 10 \text { months, } \\
\text { range ( } 4-18 \text { months) }\end{array}$ \\
\hline Hume $1991^{5}$ & $\begin{array}{l}\text { K-wire migration (1), postoperative loss of reduction } \\
\text { (TBW 53\%, PF 5\%), symptomatic metal [TBW (8)/ PF(1)], infection } \\
\text { [TBW (2)], delayed or non union [TBW (2)], heterotopic ossification } \\
{[\text { TBW (1)], ulnar neuropathy (1) }}\end{array}$ & \\
\hline Jensen $1986^{26}$ & $\begin{array}{l}\text { Sliding K-wires (24), perforated skin (10), superficial infection (1), } \\
\text { technical failure (3), failure hardware (1), subcutaneous position } \\
\text { K-wire (1) }\end{array}$ & $\begin{array}{l}\text { premature removal } 19 \\
[13 \text { weeks (range } 2-16)] \\
\text { routine removal } 13,(28 \text { weeks } \\
\text { [range } 16-52)]\end{array}$ \\
\hline
\end{tabular}

(to be continued) 
Table 6. (cont.)

\begin{tabular}{|c|c|c|}
\hline Author & Complications & Removal in \# of patients \\
\hline Karlsson $2002^{10}$ & $\begin{array}{l}\text { Radiographic arthritis (4), cysts } 25 \% \text {, subchondral sclerosis (41), } \\
\text { osteophytes (25) }\end{array}$ & 26 (TBW), 15 (Figure-of-8) \\
\hline Karlsson $2002^{24}$ & $\begin{array}{l}\text { Numbness in hand, impaired hand strength, osteoarthritis (2), } \\
\text { cysts (25), irregular subchondral bone (38), osteophytes (27) }\end{array}$ & 1 \\
\hline Koslowsky $2009^{27}$ & Infection, grade 1 or 2 arthritis & \\
\hline Kuo $2011^{9}$ & Wire migration & \\
\hline Larsen $1991^{4}$ & $\begin{array}{l}\text { Pain upon pressure (3), skin compression by cerclage wire (2), } \\
\text { pain unrelated to device (3) }\end{array}$ & 15 \\
\hline Lindenhovius $2008^{7}$ & Ulnar nerve compression, joint narrowing, arthrosis (14) & $\begin{array}{l}19 \text { (18 months, range } 6-74 \\
\text { months) }\end{array}$ \\
\hline Liu $2012^{31}$ & $\begin{array}{l}\text { CPS; skin irritation (1) TWB; implant loosening (2), proximal } \\
\text { kwire migration (4), steel wire broken (1) }\end{array}$ & 5 \\
\hline Low $1988^{32}$ & Infection (1), ulnar nerve neuropraxia(2), malunion fracture (1) & $\begin{array}{l}22 \text { (3-12 months, mean } 4.5 \\
\text { months) }\end{array}$ \\
\hline Macko $1985^{25}$ & $\begin{array}{l}\text { Prominence K-wire (15), skin breakdown proximal end K-wire (4), } \\
\text { infection soft tissue (1), K-wire migration (3), proximal end K-wire } \\
\text { bent postoperatively (4) }\end{array}$ & $\begin{array}{l}12 \text { (prominent Kwire removal, } \\
\text { range 2-6 months) }\end{array}$ \\
\hline Mullett K-Wire $2000^{12}$ & Migration hardware, infection (7) & $\begin{array}{l}19 \text { (intramedullary), } 4 \\
\text { (transcortical) }\end{array}$ \\
\hline Mullett TBW $2000^{28}$ & Prominence K-wire, skin infection, bursa formation & \\
\hline Netz $1982^{17}$ & $\begin{array}{l}\text { Subclinical skin problems (2), faulty positioning sharp cut ends } \\
\text { cerclage wires (2) }\end{array}$ & 2 (2-5 months) \\
\hline Rommens $2004^{23}$ & $\begin{array}{l}\text { Migration (8), neurolysis ulnar nerve(1), re-osteosynthesis (1), } \\
\text { delayed healing } 3.2 \% \text {, infection } 2.1 \% \text {, re-interventions } 14.7 \%\end{array}$ & 62 (average after 12 months) \\
\hline \multicolumn{3}{|l|}{${\text { Tejwani } 2002^{15}}$} \\
\hline Van der Linden $2011^{1}$ & $\begin{array}{l}\text { Short follow-up; superficial infection (7), new injury (3), transient } \\
\text { ulnar nerve neuropraxia (1), wire failure (23), gap failure (26), } \\
\text { step failure (9), elbow instability (37) } \\
\text { Long follow-up; grade } 1 \text { osteoarthritis (13), grade } 2 \text { osteoarthritis (3) } \\
\text { wire failure (10), gap failure (8), step failure (4), instability elbow (14) }\end{array}$ & \\
\hline Villanueva 2006² & $\begin{array}{l}\text { Mild residual upper limb disability, degenerative changes (10) } \\
\text { specifying in osteoarthritis grade I (3), grade II (3), grade III (4), skin } \\
\text { break down, postoperative hematoma (1), superficial infection (1) }\end{array}$ & 17 \\
\hline Wissing $1991^{20}$ & Dehiscence of fracture & 10 \\
\hline Wolfgang $1986^{33}$ & $\begin{array}{l}\text { For type IIA fractures: pain wire (2), myositis ossificans, } \\
\text { wire protrusion (2) }\end{array}$ & $\begin{array}{l}34 \text { (average after } 7.7 \text { months, } \\
\text { range } 8 \text { weeks- } 29 \text { months), } \\
25 \text { (under general } \\
\text { anaesthesia, } 12 \text { regional } \\
\text { anaesthesia) }\end{array}$ \\
\hline
\end{tabular}

ulnohumeral motion, stability and the ability to perform five functional tasks in daily life. The total score ranges from 5 to 100 , with higher scores indicating better function. A total score is classified excellent between 90 and 100 points, good between 75 and 89 points, fair between 60 and 74 points and poor at less than 60 points $^{34}$.

The Disability of Arm, Shoulder and Hand (DASH) questionnaire consists of three parts where each item is ranked in a five-point scale. Questions about symptoms and disabilities of the upper limb are considered in the first part. The other two parts are optional and include sports, music and work activities. The scores of all parts are summed up and transformed in the DASH score, which ranges between 0 (no disability) to 100 (severe disability) ${ }^{35}$.

\section{Discussion}

In 1982, Netz and Stromberg ${ }^{17}$ introduced non-sliding pins to overcome one of the most occurring complications, backing out of the K-wires, which leads to skin problems. They were convinced that the commonly used K-wires should be exchanged for the non-sliding Netz pins. However, no complications are also ex- 
pected when the free ends of the cerclage wires are threaded through the loops on the medial or lateral side of the ulna ${ }^{17}$. Jensen et al. ${ }^{26}$ focused on the drawbacks of TBW, which were experienced by 29 of 55 patients, with skin troubles in 24 patients being the most frequent. They also suggested minor operative modifications such as burying the ends, preferably under the muscle on the radial side of the ulna to reduce complications. They also used the non-sliding (Netz) pins, to overcome the problem that the drill hole made by the K-wire has a tendency to enlarge. The non-sliding pin was also assessed by Larsen et al. ${ }^{4}$ in combination with TBW. In this case the nonsliding pin also eliminates pin migration outside the bone, as well as migration into the intramedullary canal. This technique does not cause discomfort from the metallic device, so this may be left in place avoiding a second operation.

In 1987 Wolfgang et al. ${ }^{33}$ advised using TBW for isolated olecranon fractures. True K-wire migration was not common, and was probably eliminated by carefully burying the ends and exact surgical technique. Supplemental fixation must be used when necessary. Holdsworth et al. ${ }^{3}$ also believe that TBW is a reliable method for all patterns of olecranon fracture. In their investigation, $56 \%$ of the time the wires were removed, half of the time patients did not complain but routine removal had been suggested. Range of motion data confirmed that loss of extension is much more frequent than loss of flexion. In 1985, Macko et al. ${ }^{25}$ reported several complications using TBW. Not only prominence of the K-wires leading to pain at the insertion site or skin break down, but also other minor complications are attributable to surgical technique. They recommended removing the hardware, which was undertaken for most elbows 3 months postoperatively, when radiographic union had been achieved. Rommens et al. ${ }^{23}$ also report that patients treated with different forms of TBW more often expressed subjective complaints and loss of function in activities of daily life before metal removal. Therefore they recommend removing the metal after fracture healing. After 1 year, fracture healing is advanced enough to resist traction forces of the elbow in flexion and extension. In 1988, Low et al. ${ }^{32}$ also concluded that TBW is the ideal method for internal fixation of olecranon fractures. Early mobilization allows the return to full range of motion without affecting union. Drawbacks commonly associated with TBW were not observed during this study. 20 years later, TBW fixation remains the "gold standard" for treatment for both of displaced and minimally comminuted olecranon fractures $^{21}$. In the long term, low levels of pain and elbow degenerative changes were evident, but no clear association could be established between radiological and clinical results ${ }^{19}$. The TBW technique was also thoroughly studied by Villanueva et al. ${ }^{2}$ : hardware removal was required in 17 of 37 patients. Nonetheless, TBW provides satisfactory results in a high percentage of fractures. One remark is that outcome may be compromised in the presence of elbow instability and associated fractures of the radial head and coronoid.
Although isolated simple transverse olecranon fractures seem to do well with a variety of treatment modalities, more complex fractures require specific reconstruction techniques.

While non-sliding pins were used by Netz, Jensen and Larsen to overcome the problem of sliding of the $\mathrm{K}$ wires inducing skin problems, careful use of the techniques burying ends of K-wires diminishes the complications. TBW still seems to be an ideal method for treatment, but hardware removal after union is advised.

\section{Comparing configurations and techniques}

For TBW with K-wires, in general two configurations are compared, namely intramedullary and transcortical. Ahmed et al. ${ }^{13}$ compared two different methods on transverse or oblique olecranon fractures. They concluded that the group with intramedullary cancellous screws and tension band had better clinical results compared to the AO TBW group, regarding pain, function and range of motion. Within the AO TBW group no significant difference between transverse or oblique fractures was found. Huang et al. ${ }^{6}$ focused on different configurations of K-wires; the locations of the ends varied between the proximal ulnar canal, through the anterior ulnar cortex and in the distal ulnar canal. Each of these three methods achieved a high success rate. Nevertheless, insertion of the K-wires in the proximal ulnar canal has to be avoided as it is associated with the higher rate of backing out of the wires and irritation of the skin around the elbow, and exhibits the poorest stability. TBW is an excellent technique for treating olecranon fractures of all types, and the ends of the K-wires should be placed into the distal ulnar canal to increase stability. Kuo et al. ${ }^{9}$ also tried to solve the high incidence rate of skin complications by studying the optimal fixation of TBW. Two groups were compared, a bicortical K-wire group and an intramedullary group. Radiographic union was achieved in both groups. However, migration of the Kwires was higher in the intramedullary group than in the bicortical group. Recommendations are that the bicortical method can be applied in articular fractures extending to the distal part of the ulna. This avoids damage to the neurovascular bundle and cartilage of the olecranon, while the intramedullary method can be applied in the group including transverse and articular fractures extending to the proximal part of the ulna. In this case, the K-wires should be buried beneath the triceps. Van der Linden et al. ${ }^{1}$ also investigated two different configurations for K-wires, intramedullary and transcortical. Instability was found in $78 \%$ of the patients treated with intramedullary K-wires, compared to $36 \%$ of the patients with transcortical K-wires. When instability occurs, the tendency is to develop more osteoarthritis. Functional outcome was better for patients in whom the hardware was removed. Therefore, transcortical K-wires are recommended so local complications can be minimized and a better longterm outcome can be expected. When patients show a limited range of motion, removing the metal work is 
advised. When comparing the transcortical and intramedullary configurations, Mullett et al. ${ }^{12}$ also found that both techniques are very successful for internal fixation of transverse olecranon fractures. However, they also found that there is a high rate of local complications when using the intramedullary canal configuration, while the transcortical method shows far lower complication rates and five times less implant removal is necessary. On radiographs, the rate of wires backing out was three times greater for patients in whom the K-wires followed the long axis of the ulna, rather than across the anterior cortex. Mullet et al. ${ }^{28}$ demonstrated, in 34 transverse olecranon fractures in patients older than 70 , that the transcortical method of TBW is most successful, avoiding local complications. When focusing on the elderly, post-operative complications should be avoided. They suggest the routine use of this technique to reduce the morbidity associated with treatment of this common injury.

Overall, the transcortical method of K-wire insertion results in the best outcome, leading to less instability and therefore less chance of osteoarthritis ${ }^{1}$, less sliding of the pins so less prominence of hardware ${ }^{9}$ and an overall lower rate of complications and less implant removal necessary ${ }^{12}$. Furthermore, especially in the elderly, reoperation should be avoided so the use of transcortical K-wires is advised ${ }^{12,28}$.

Fan et al. investigated three different techniques; all achieved a high union rate within an average of 3.4 months. The olecranon screw and K-wire group achieved higher satisfaction than the rush pin group, but without significant difference. The few complications that occurred in the rush pin and K-wire group were not difficult to manage. For olecranon fractures with a small fragment, they advise to use K-wires.

Karlsson et al. ${ }^{10}$ compared figure-of-eight wiring with TBW at a mean of 18 years after injury. Removal of the hardware was performed in $81 \%$ of the patients for TBW, and in $43 \%$ of the patients having figure-ofeight wiring. In this study no differences were found between the two techniques. Degenerative joint changes were found in more than half of the fractured elbows, but few developed clinically evident osteoarthritis. This outcome was not different between the groups. However Karlsson et al. recommend using figure-of-eight wiring, as the need for hardware removal is $50 \%$ less.

Lindenhovius et al. ${ }^{7}$ also studied the long-term outcome with different types of treatment was done by: one of four $1 / 3$ tubular plates failed. These plates are no longer considered of adequate strength for any type of olecranon fracture. Of the 20 patients, $7 \mathrm{de}$ veloped an ulnar neuropathy. Only two of these patients were identified and treated operatively. Lindenhovius et al. concluded that the initial post-operative results are durable in the long term. Pain, decreased final range of motion and ulnar neuropathy were the most important predictors of functional outcome and results. Rommens et al. ${ }^{23}$ showed a statistically significant association between fracture pattern and development of arthrosis and between suboptimal osteosynthesis and arthrosis. However, this was more often seen in multifragmentary fractures than in simple fractures.

A long-term study ${ }^{24}$ with an average of 19 years follow-up, showed that $84 \%$ of patients reported no complaints, and $4 \%$ still experienced daily pain. Radiographic signs of degenerative changes were found in more than $50 \%$ of the formerly fractured elbows, while this was $11 \%$ in the control of uninjured elbows. Older individuals might need a longer rehabilitation period. The reoperation rate was $48 \%$ in this study. Long-term follow-up studies suggest that initial results after operation are durable over the long-term. Degenerative changes are observed in long-term follow-up studies, but this does not immediately indicate that osteoarthritis develops. Fracture pattern and suboptimal osteosynthesis do seem to correlate with arthrosis, but are more frequent in multifragmentary fractures.

\section{Plate fixation techniques}

"Plate fixation should be strongly considered when treating displaced olecranon fractures" (Hume et al. $)^{5}$. They compared TBW and PF and showed that PF is superior to TBW, as prominence of the metal was frequently observed in TBW (42\%) and at follow-up an articular step-off or gap was more frequently observed in TBW (53\% compared to $5 \%$ in PF). In 5 of 11 transverse olecranon fractures the gap or step-off was equal or greater than $2 \mathrm{~mm}$ when using the TBW technique, compared to PF were there was none observed for this fracture type. Clinical and roentgenographic results were also better in PF. One drawback is that PF requires an average of 25 minutes longer operation, but this did not lead to postoperative complications.

A variant of PF using congruent anatomic elbow plate fixation ${ }^{14}$ seems safe and effective for different types of olecranon fractures. This technique was assessed only on a small number of patients, and showed low rates of hardware removal and enough stability for early motion. Bailey et al. ${ }^{16}$ also used plate fixation for comminuted and simple olecranon fractures. All the goals of olecranon fracture management were met; physical capacity measures returned to premorbid function, forearm supination showed small but significant decrease in motion. A minimal loss of physical capacity, little pain and disability, normal health and high patient satisfaction were observed. No differences in outcome between Mayo Type II and III fractures were found. Tejwani et al. ${ }^{15}$ also studied a more innovative approach using a posterior hook plate. This technique showed significantly less displacement compared to the TBW technique. All fractures united, and no hardware related problems occurred. This posterior plating achieves greater stability, is universal for all fracture types and has a low profile of the implant. This technique is believed to lead to fewer reoperations for removal of the hardware ${ }^{15}$.

Overall, different forms of plate fixation techniques seem to be a good option for treatment of all fracture 
types, as complications seem limited. Therefore, removal or reoperation is reduced, and clinical and roentgenographic results are satisfying.

\section{Other techniques}

Koslowsky et al. ${ }^{27}$ tested two different implants for reconstruction of the olecranon; fine-threaded wires (FFS) with a washer versus K-wires, both with a low rate of hardware related problems. They paid extra attention to the length of the proximal implants to prevent possible skin irritation and local pain. An accompanying biomechanical study also indicates that the FFS system gives good stability without additional TBW and could be an alternative treatment for displaced olecranon fractures.

Wissing and van der Werken 20 focused on absorbable material Vicryl No 2. With this method no skin problems were observed in 36 patients with different fracture patterns.

Liu et al. ${ }^{31}$ compared a cable pin system (CPS) to TBW. They describe their technique as following; "The CPS is a new design, according to the principles of TBW, that combines a partially threaded $4.0 \mathrm{~mm}$ cancellous lag screw (pin) with a cable. After inserting the partially threaded pin portion into the bone, the cables are wrapped externally around the bone and tension is applied. The cable attached to the pin prevents the pin from backing out, creates compression at the fracture site and is prevented from migrating. Biomechanical properties of the cable make it superior to wire. Cable has a better static strength and much greater fatigue strength than monofilament wire, in addition cable is more flexible which makes it easier to insert and remove and less irritating to the bone surface and soft tissues than wire"31. Their results showed that for TBW fracture healing, postoperative complications and elbow function were associated with a significantly lower rate of excellent results. Slower healing time was also associated with patient age. Compared to TBW the CPS shows better healing time, less postoperative complications and a higher MEPS. On the other hand, the technique is costly and has some shortcomings in pin length; also it is not suitable for multifragmentary fractures. This is an effective and reliable method which is worthy of clinical application ${ }^{31}$.

\section{Children}

Olecranon fractures in children are rare, and, when treating them, care has to be paid as the fracture involves the olecranon growth plate. Impairment of blood supply to the growth plate must be prevented by balancing between a sufficiently stable fixation and being minimally invasive. Gicquel et al. ${ }^{30}$ present preliminary results of a fixation technique using threaded pins with an adjustable lock. The study only considers six patients: five have an excellent clinical grading, and one good. The pins were removed early after 6 weeks. They cannot conclude the technique is safe, but it fulfills the criteria which are necessary in children. Gortzak et al. ${ }^{19}$ also performed a study in children, applying open reduction and fixation with percutaneously placed K-wires and absorbable tension band suture. In all six patients the wires were removed after four weeks, stable fixation and union was achieved. Subsequent reoperation was avoided using absorbable sutures (Vycril, Dexon 11 or PDS II).

Hope et al. ${ }^{18}$ compared biodegradable polyglycolic acid (PGA) pins with standard K-wires in elbow fractures of children, with only two having olecranon fractures, both assigned to different groups. In general, the K-wire group exhibited adequate fixation, but three children developed infections and one developed ectopic bone. Most of the removals took place under general anaesthesia as the wires were fixed too firmly and the younger children would not tolerate local anesthesia. The PGA pins have the advantage that they do not need to be removed and also provide satisfactory union. However, PGA pins can cause an inflammatory response which does not appear to interfere with fracture healing. Hope et al. used thinner PGA pins so less material degrades, which may prevent a local inflammatory response. As these pins do not require removal, this saves an operation, and are a suitable alternative to K-wires.

Arbes et al. ${ }^{29}$ compared a variety of methods in 15 children. From a clinical point of view, all methods achieved satisfactory results, while the imaging results were less satisfactory. The quality of postoperative reduction seems to be related to these observations. Four patients received absorbable tension band sutures and provided fixation equal to that of TBW. Arbes et al. also found that this technique is associated with less and easier hardware removal and therefore advise it, leading to decreased local complications while providing excellent fixation ${ }^{29}$.

All four studies include a small patient population to show clinical and radiographic significance.

\section{Limitations and strengths}

Strengths of the present systematic review were the large amount of studies considered with no limitations to time. This shows that TBW was already advised in the 1980s, and is still the most commonly used method. Given the comprehensive search method, we identified a large number of articles which would not have been found using only one source. Selection biases were diminished, as the selection was undertaken by two independent reviewers.

The goal was to give an overview of the current methods applied. This review shows several possible treatment options with varying successful outcome. Furthermore, the use of the CMS score to rate articles is a good way to assess the quality of the studies: in this case, it gave a clear indication that the overall quality was relatively poor.

This study also has several limitations. Most articles identified included not just displaced transverse ole- 
cranon fractures, and also discussed other fracture types, with only two dealing exclusively with the fracture type of interest. The distinction between the different types of fracture and their outcome was usually not well stated. Furthermore, we acknowledge that assessing CMS score only once is possibly a limitation of the present study. However, the CMS score of the two investigators did not differ much, and consensus was easily reached. Nevertheless, it would be better to assess the articles twice to obtain inter- and intra observer reliability. This will probably not change the fact that the overall score of the selected studies was low. Direct comparison of different forms of internal fixation is hard, as postoperative care differs and is not described in every article.

\section{Recommendations and conclusions}

The overall CMS score was poor, as not all articles provide sufficient data. The goal of treatment of displaced olecranon fractures is to restore early, active, elbow motion and prevent joint stiffness. Therefore, it is necessary to use an unambiguous classification score of type of fractures. Also, generating results of treatment requires comprehensive methods that can assess the elbow function. Time to union and elbow function are poorly described. Furthermore, different fracture types are assessed, and therefore discussing specifically transverse displaced olecranon fractures is impossible. Another issue is to take into account arm dominance. So far, the results are usually discussed according to the technique used, while it is suggested that simple fractures need other treatments than more complex ones. In the end more patients and longer follow up are necessary. Most studies were retrospective: such a study design is more prone to selection bias and is sensitive to misclassification, but such investigations allow to analyse multiple outcomes at the same time. As restoring elbow function is the goal of treatment one would expect that elbow function is clearly described in the results. However, not all articles describe symptoms, range of motion and radiographic and clinical outcomes.

TBW is still the most widely applied method to operatively manage olecranon fractures, with the transcortical method of using K-wires the most satisfactory. Plate fixation is a good alternative as complications are minimal. Other techniques using absorbable sutures are less investigated, but are promising, especially in children.

The study has been conduct ethically according to international standards and as required by the journal ${ }^{36}$.

\section{References}

1. van der Linden SC, van Kampen A, Jaarsma RL. K-wire position in tension-band wiring technique affects stability of wires and long-term outcome in surgical treatment of olecranon fractures. Journal of shoulder and elbow surgery/American Shoulder and Elbow Surgeons.2012;21:405-411.
2. Villanueva P, Osorio F, Commessatti M, Sanchez-Sotelo J. Tension-band wiring for olecranon fractures: analysis of risk factors for failure. Journal of shoulder and elbow surgery/ American Shoulder and Elbow Surgeons. 2006;15:351-356.

3. Holdsworth BJ, Mossad MM. Elbow function following tension band fixation of displaced fractures of the olecranon. Injury. 1984;16:182-187.

4. Larsen $\mathrm{E}$, Jensen $\mathrm{CM}$. Tension-band wiring of olecranon fractures with non sliding pins. Report of 20 cases. Acta orthopaedica Scandinavica. 1991;62;360-362.

5. Hume MC, Wiss DA. Olecranon fractures - a clinical and radiographic comparison of tension band wiring and plate fixation. Clinical Orthopaedics and Related Research. 1991;229-235.

6. Huang TW, et al. Tension band wiring for olecranon fractures: relative stability of Kirschner wires in various configurations. The Journal of trauma. 2010;68:173-176.

7. Lindenhovius ALC, Brouwer KM, Doornberg JN, Ring DC, Kloen P. Long-term outcome of operatively treated fracturedislocations of the olecranon. Journal of orthopaedic trauma. 2008:22:325-331.

8. Morrey BF. Current concepts in the treatment of fractures of the radial head, the olecranon and the coronoid. Journal of Bone and Joint Surgery. 1995;77A:316-327.

9. Kuo CC, Hsu HC, Hong SW, AI E. Biomechanical assessment of tension-band wiring for olecranon fractures. Biomedical Engineering: Applications, Basis and Communications. 2011;23: 83-87.

10. Karlsson MK, Hasserius R, Besjakov J, Karlsson C, Josefsson $\mathrm{PO}$. Comparison of tension-band and figure-of-eight wiring techniques for treatment of olecranon fractures. Journal of shoulder and elbow surgery/American Shoulder and Elbow Surgeons. 2002;11:377-382.

11. Fan $\mathrm{G}, \mathrm{Wu} \mathrm{CC}$, Shin $\mathrm{CH}$. Olecranon fractures with tension band wiring techniques - comparison among three different configurations. Chang Gung Medical Journal. 1993;16:231238.

12. Mullett $\mathrm{JH}$, et al. K-wire position in tension band wiring of the olecranon - a comparison of two techniques. Injury, Int J Care Injured. 2000;31:427-431.

13. Ahmed AR, Sweed T, Wanas A. The role of cancellous screw with tension band fixation in the treatment of displaced olecranon fractures, a comparative study. European Journal of Orthopaedic Surgery \& Traumatology. 2008;18:571-576.

14. Anderson ML, Larson N, Merten SM, Steinmann SP. Congruent elbow plate fixation of olecranon fractures. Journal of orthopaedic trauma. 2007;21:386-393.

15. Tejwani NC, Garnham IR, Wolinsky PR, Al E. Posterior Olecranon Plating. Hospital for Joint Diseases. 2003;61:27-31.

16. Bailey CS, MacDermid J, Patterson SD, King GJ. Outcome of plate fixation of olecranon fractures. Journal of orthopaedic trauma. 2001;15;542-548.

17. Netz $P$, Strömberg L. Non-sliding pins in traction absorbing wiring of fractures: a modified technique. Acta orthopaedica Scandinavica. 1982;53:355-360.

18. Hope PG, Williamson DM, Coates CJ, Cole WG, Clinical I. Biodegradable pin fixation of elbow fractures in children. Journal of Bone and Joint Surgery. 1991;73B:6-9.

19. Gortzak Y, Mercado E, Atar D, Weisel Y. Pediatric Olecranon Fractures. Journal Pediatric Orthopedics. 2006;26:39-42.

20. Wissing JC, Van der Werken C. Die Zuggurtungsosteosynthese aus resorbierbarem Material. Unfallchirurg. 1991;94:45-46.

21. Chalidis BE, Sachinis NC, Samoladas EP, Dimitriou CG, Pournaras JD. Is tension band wiring technique the "gold standard" for the treatment of olecranon fractures? A long term functional outcome study. Journal of orthopaedic surgery and research. 2008;3:9.

22. Weber BG, Vasey H. Osteosynthesis in olecranon fractures. $Z$ Unfallmed Berufskr. 1963;56:90-96. 
23. Rommens PM, Küchle R, Schneider RU, Reuter M. Olecranon fractures in adults: factors influencing outcome. Injury. 2004; 35;1149-1157.

24. Karlsson MK, Hasserius R, Karlsson C, Al E. Fractures of the olecranon - a 15- to 25-year followup of 73 patients. Clinical Orthopaedics and Related Research. 2002;403:205-212.

25. Macko D, Szabo RM. Complications of tension-band wiring of olecranon fractures. Journal of Bone and Joint Surgery. 1985; 67A:1396-1401.

26. Jensen CM, Olsen BB. Drawbacks of traction-absorbing wiring (TAW) in displaced fractures of the olecranon. Injury. 1986;17:174-175.

27. Koslowsky TC, et al. Olecranon fracture fixation with a new implant: biomechanical and clinical considerations. Injury. 2009;40;618-624.

28. Mullett H, Noel J, Shannon F, O'Rourke SK. European Joumal of Tension band wiring of transverse olecranon fractures in the elderly-transcortical K-wires prevent backout. European Journal of Orthopaedic Surgery \& Traumatology. 2000;10;65-67.

29. Arbes $S$, Platzer P, Vécsei V. Surgical treatment of olecranon fractures in children. European Journal of Orthopaedic Surgery \& Traumatology. 2011;22:209-212.
30. Gicquel P, Giacomelli MC, Karger C, Clavert JM. Surgical technique and preliminary results of a new fixation concept for olecranon fractures in children. Journal of pediatric orthopedics. 2003;23:398-401.

31. Liu QH, et al. Randomized prospective study of olecranon fracture fixation: cable pin system versus tension band wiring. The Journal of international medical research. 2012;40:10551066.

32. Low CK, Low BY. Olecranon fracture and tension band wiring Sing Med J. 1988;29:480-484.

33. Wolfgang G, Burke F, Bush D, et al. Surgical treatment of displaced olecranon fractures by tension band wiring. Clinical Orthopaedics and Related Research. 1987;223:192-204.

34. Morrey BF, An KN, Chao EY. Functional evaluation of the elbow. The elbow and its disorders. 1993;20:86-89.

35. Jester A, Harth A, Germann G. Measuring levels of upper-extremity disability in employed adults using the DASH Questionnaire. The Journal of hand surgery. 2005;30;1074.e11074.e10.

36. Padulo J, Oliva F, Frizziero A, Maffulli N. Muscles, Ligaments and Tendons Journal. Basic principles and recommendations in clinical and field science. MLTJ. 2013; 4:250-252. 